\title{
External influence
}

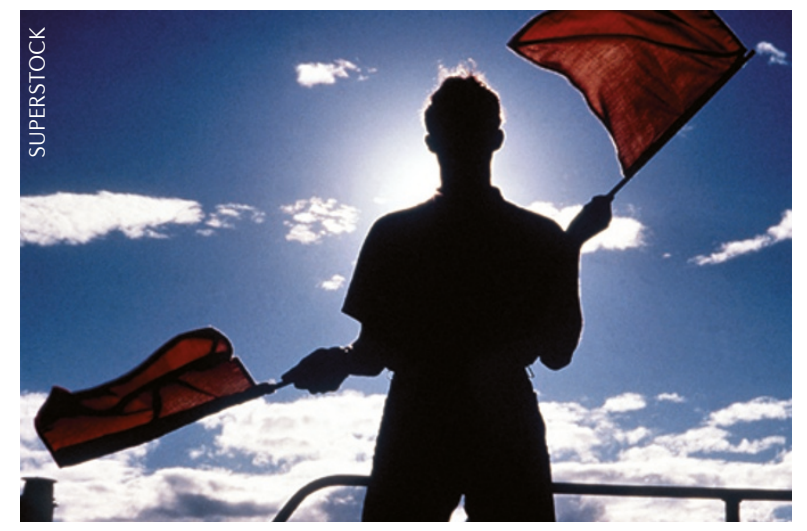

Maintaining the balance between protein synthesis, folding and clearance is typically regarded as a cell-autonomous process. Disruption of protein homeostasis can lead to the formation of toxic protein aggregates, such as those associated with Huntington's disease and amyotrophic lateral sclerosis. Morimoto and colleagues now demonstrate the existence of a non-cell-autonomous modulation of this delicate process.

Polyglutamine (PolyQ)-containing proteins are particularly prone to forming aggregates and causing cellular toxicity. The authors of this study carried out a forward genetic screen of Caenorhabditis elegans strains expressing PolyQ fused to yellow fluorescent protein, to identify mutants with premature PolyQ aggregation in muscle cells. They found that a mutation in the DNA-binding domain of $u n c-30$, which encodes a neuron-specific regulator of GABA ( $\gamma$-aminobutyric acid) synthesis, not only causes defective GABAergic signalling but also leads to the premature conversion of soluble PolyQ to an aggregated form in muscle cells and to an uncoordinated phenotype. Two known unc-30-null alleles and mutations in two UNC-30 targets (unc-47 and $u n c-25)$ gave rise to a similar phenotype, indicating that defective inhibitory GABA signalling in motor neurons can affect protein aggregation in post-synaptic muscle cells.

To determine whether this effect was due to overstimulation of muscle cells by excitatory acetylcholine (ACh) inputs, Morimoto and colleagues examined acetylcholinesterase-mutant worms. These worms are known to exhibit muscle hypercontraction due to defective clearance of ACh from the synaptic cleft; interestingly, they were found to exhibit a premature aggregation of PolyQ. ACh increases $\mathrm{Ca}^{2+}$ influx into muscle cells, and an even more dramatic PolyQ aggregation was observed in worms carrying a gain-of-function mutation in the gene encoding the $\alpha 1$ subunit of the $\mathrm{Ca}^{2+}$ channel.
Finally, to investigate whether the unc-30 mutation causes a general disruption of protein homeostasis, the authors investigated the effects of mutant UNC-30 on the homeostasis of metastable structural proteins containing temperature-sensitive (ts) mutations that cause defective folding, leading to uncoordinated movement or paralysis at the restrictive temperature. Worms carrying both the unc-30 and the ts mutation exhibited the ts phenotype at permissive temperatures, further suggesting that muscle overexcitation compromises the folding of proteins that have mild folding defects or are aggregation prone.

The exact mechanism whereby intercellular communication affects protein homeostasis remains to be determined. However, if this process turns out to be a common feature of protein conformation disorders, it could have wide-ranging implications for the development of therapies for, and the prevention of, both neuromuscular and neurodegenerative diseases.

\section{Monica Hoyos Flight}

ORIGINAL RESEARCH PAPER Garcia, S. M., Casanueva, M. O., Silva, M. C., Amaral, M. D. 8 Morimoto, R. I. Neuronal signaling modulates protein homeostasis in Caenorhabditis elegans post-synaptic muscle cells. Genes Dev. 21, 3006-3016 (2007) 